\title{
Performance Control of Semiconductor Optical Amplifier and Fiber Raman Amplifier in Communication System
}

\author{
Roby Ikhsan ${ }^{\S}$, Romi Fadli Syahputra ${ }^{\S}$, Saktioto ${ }^{\S}$, Okfalisa \\ $\S$ Department of Physics, Mathematics and Natural Sciences, Universitas Riau \\ Kampus Bina Widya, Simpang Baru, Panam km 12.5, Pekanbaru, Indonesia \\ " Department of Science and Technology, Universitas Islam Negeri Suska, Pekanbaru, Indonesia \\ Corresponding author: saktioto@ieee.org
}

\begin{abstract}
Optical fiber communication greatly develops considering the limitations of communication systems corresponding to the demand of information needs. Optical fiber is one of waveguides that is able to transmit information with large capacity, high speed and low attenuation. Due to the very long distance, then the signal transmission needs to be maintained from the disturbance of geometry, materials and signal sources. To maintain the signal, optical amplifier is required although several amplifiers still have problems. This paper investigates the performance of Semiconductor Optical Amplifier (SOA) and Fiber Raman Amplifier (FRA). Using the simulation application, both amplifiers are designed and compared by inline amplifiers. The results showed that the transmission distance of FRA is more stable than SOA. However, this FRA system has higher power consumption when compared to the SOA system.
\end{abstract}

Keywords-fiber optic, SOA, FRA, optical amplifier

\section{INTRODUCTION}

Optical transmission media greatly affect the performance of a communication system. Fiber optic is one of transmission media which is capable of transmitting information with a large capacity, high speed and has low attenuation. Although optical fiber provides many advantages but there are also disadvantages that can disrupt the performance of the fiber optics, the effects that can limit the delivery and speed of data transmission. This effect is divided into linear effects and nonlinear effects. Linear effects include attenuation and dispersion; a distortion in the beam of light passing through the optical fiber core caused by different modes, wavelengths and velocities. While non-linear effects arise due to Kerr effect in the form of Self Phase Modulation (SPM), Cross Phase Modulation (XPM) and Four Wave Mixing (FWM) and the result of Inelastic Scattering including Stimulated Raman Scattering (SRS) and Stimulated Brillouin Scaterring (SBS) [1]. These linear and nonlinear effects can damage information signals such as widespread light pulses, reduced bandwidth shortened, transmission distance and limited bit rate. All this is a communication disorder.

The suitable amplifier is one way to deal with the effects of linear and non-linear disturbances as well as maximize the working of optical transmission media. Generally the optical amplifier consists of Semiconductor Optical Amplifier (SOA), Fiber Raman Amplifier (FRA) and Erbium-Doped Fiber Amplifier (EDFA). SOA is designed in the form of Quantum Dot SOA network as linear network and Bulk SOA as nonlinear network [2]. Then, it is known that SOA has a high non-linear nature, low power consumption, fast operating speed, and can easily be used in photonic systems [3-6].

In SOA type amplifier, the gain can be calculated using the following equation:

$$
g_{m}=A_{g}\left(N-N_{0}\right)
$$

where

$g_{m}:$ material amplifier

$A_{g}:$ coefficient of derivative gain

$N$ : carrier density

$N_{0}$ : carrier density at the point of transparency

$$
g_{t}=\Gamma g_{m}-\alpha
$$

where

$g_{t}:$ coefficient of amplifier

$\Gamma$ : optical confinement factor

$\alpha$ : effective loss coefficient

$$
G=\exp \left(g_{t} z\right)
$$

where $G$ is magnitude of gain $(\mathrm{dB})$ and $z$ is length of optical fiber $(\mathrm{m})$.

Then Bromage introduced the Raman amplifier used in fiber optic communication systems [7]. The gain on this amplifier can be calculated using the equation as follows,

$$
\begin{gathered}
G=10 \log (\exp (g P L)) \\
g=2 \gamma \rho \operatorname{Im}\left(\chi_{1111}\left(\omega_{p}-\omega_{s}\right)\right)
\end{gathered}
$$




$$
\gamma=\frac{2 \pi n_{2}}{\lambda A_{e f f}}
$$

where

$P$ : power pump (Watt)

$L$ : length of optical fiber (m)

$g$ : Raman gain coefficient

$\gamma:$ non-linear phase change (rad)

$\rho:$ non-linear polarization fraction

$\chi_{1111}\left(\omega_{p}-\omega_{s}\right):$ Raman's susceptibility

$n_{2}$ : non-linear refractive index $\left(\mathrm{m}^{2} . \mathrm{W}^{-1}\right)$

$A_{\text {eff }}$ : effective surface $\left(\mathrm{m}^{2}\right)$

$\lambda$ : wavelength signal (m)

Both amplifiers show that the type of SOA more considered the carrier density and the material factor, whereas FRA more considered the frequency characteristics and wave non-linear conditions. However, both amplifiers are very dependent on the media passed by the signal. In order to investigate the performance SOA and FRA, bit error rate (BER) and Q-factor are two parameters used to measure their characteristics.

BER and Q-factor is one of the most important factors that limiting the transmission distance in optical communication system. In order to transmit signals over long distances, it is necessary to have a low BER and high Q-factor within the fiber. The optical amplifier in the fiber represents the optical signals to be directly amplified optically without any conversion. The BER is an indication of how often data has to be retransmitted because of an error. Too high a BER may indicate that a slower data rate would actually improve overall transmission time for a given amount of transmitted data since the BER might be reduced, lowering the number of packets that had to be present. Bit error rate (BER) as the number of detected bits that are incorrect before error correction, devided by the total numberof transferred bits (including redundant error codes). Normally, the transmission BER is larger than the information BER. The information BER is affected by the strength of the forward error correction code. There are kinds of BER occurring in a communication system. It may be affected by transmission channel noise, interference, distortion, bit synchronization problems, attenuation, wireless multipath fading, etc. However, in both amplifiers, we consider as the simple channel model and data source model.

Another factor is Q-factor, a parameter that describes the resonance behavior of an underdamped harmonic oscillator (resonator). Sinusoidally driven resonators having higher Q factors resonate with greater amplitudes (at the resonant frequency) but have a smaller range of frequencies around that frequency for which they resonate; the range of frequencies for which the oscillator resonates is called the bandwidth. High-Q oscillators oscillate with a smaller range of frequencies and more stable. Q-factor is dimensionless parameter that describes how underdamped an oscillator or resonator is and characterizes a resonator's bandwidth relative to its center frequency.

\section{RESEARCH METHOD}

\section{A. Design and Preparation of Optical Models}

The design of the network model will be simulated using Optisystem version 11.1.0.53. In the model, the SOA and FRA optical amplifiers are coupled to the transmission amplifier network (inline amplifier). Figure F.1 shows the design of model, optical fiber communication system.

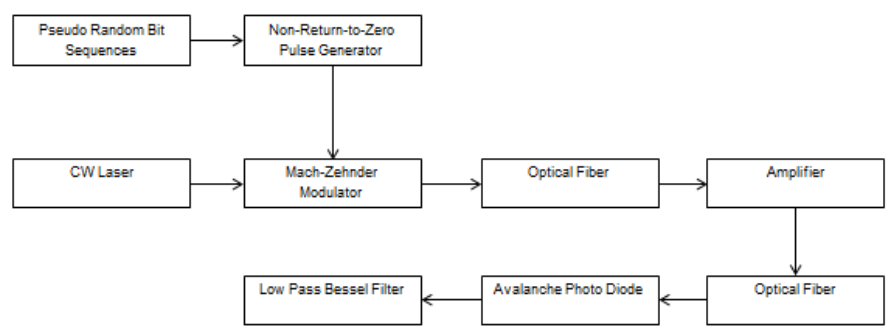

Figure F.1. Model Design with Amplifier

a. Information Source Block

The information source block consists of two components: Pseudo-Random Bit Sequence (PRBS) and Non-Return-toZero Pulse Generator (NRZ). PRBS functions to generate bits with specific patterns and speeds. Then the bit that has been generated by PRBS will be encoded using NRZ coding technique. NRZ coding technique has the advantage, that is more resistant to noise and is not affected by the voltage level. Figure F.2. is the planning drawing for the source of pulse information.

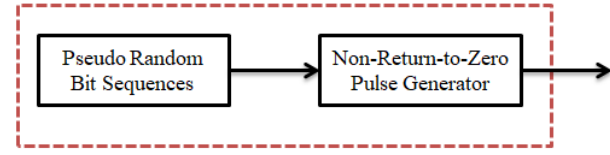

Figure F.2. Information Source Block

\section{b. Transmitter Block}

The Transmitter Block (Figure F.3) consists of two components: the laser and the modulator. The laser acts as a light signal generator on a network system. The type of laser used is a Continuous Wave (CW) laser. The modulator used is the Mach-Zehnder Modulator (MZM) which will modulate the coded information signal with the output signal laser.

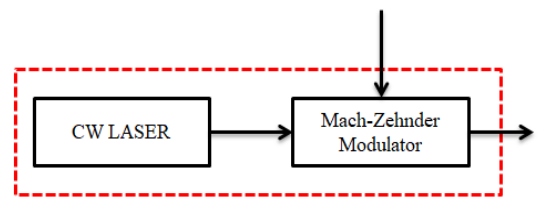

Figure F.3. Information Source Block

\section{c. Transmission Media Block}

The transmission medium (F.4) on this optical network uses single mode optical fiber, because it has a wide bandwidth and a considerable range. In order for the transmitted signal to reach a considerable distance then it requires an optical amplifier, SOA and FRA. Type of TRD used is Average Power Model Amplifier (APA). 


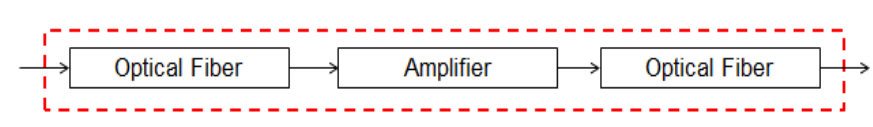

Figure F.4. Information Source Block

\section{d. Receiver Block}

The Receiver block consists of two components: a detector and a filter. The detector used is Avalanche Photo Diode (APD) as shown in Figure F.5, because it has a faster response and higher gain. The already converted information signal will be forwarded to the filter. The working principle of this filter passes a certain frequency and dampens other frequencies. The type of filter used is the Low Pass Bessel Filter.

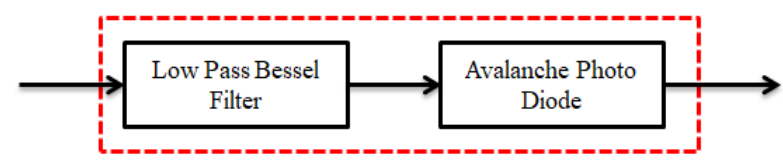

Figure F.5. Information Source Block

\section{B. Work Procedures of SOA and FRA Design}

The stages of this procedure begin with a preliminary simulation process that aims to determine the maximum transmission distance of signal propagation on the optical fiber without any gain. In the simulation process it will iterate on the optical fiber, for 30 iterations and each iteration is $10 \mathrm{~km}$. Then, the determination of maximum transmission distance is demonstrated by using SOA and FRA. The working procedure of these two optical amplifiers can be seen in Figure F.6 and F.7.

\section{Result and Simulation of Bit Error Rate and Q-Factor}

The length of fiber optics greatly affects the performance of a communication system. In determining the maximum transmission length, the parameters that play an important role are the Bit Error Rate (BER) and Q factor. According to the rules of the International Telecommunications Union (ITU-T G.691; ITU-T G.692; ITU-T G.693), the BER requirements for optical communication systems must be better than $10^{-12}$, meaning that the minimum value of BER system should be smaller from $10^{-12}$. Q-factor is a quality factor that will determine the quality of a link. In a fiber optic communication system, the minimum size of a good Q-factor is 6 .

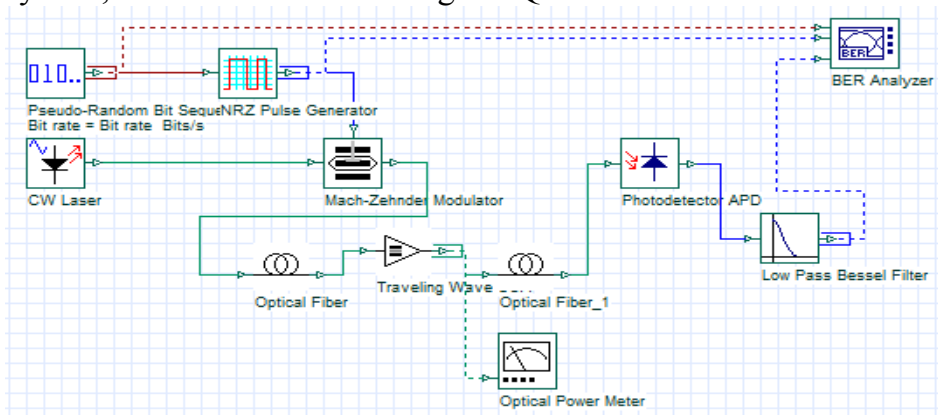

Figure F.6. Optical Fiber System Scheme with SOA InlineAmplifier

The power consumption of the amplifier will be measured using the Optical Power Meter contained in the circuit. Then we will also see the influence of the wavelength on the maximum transmission distance of the system.

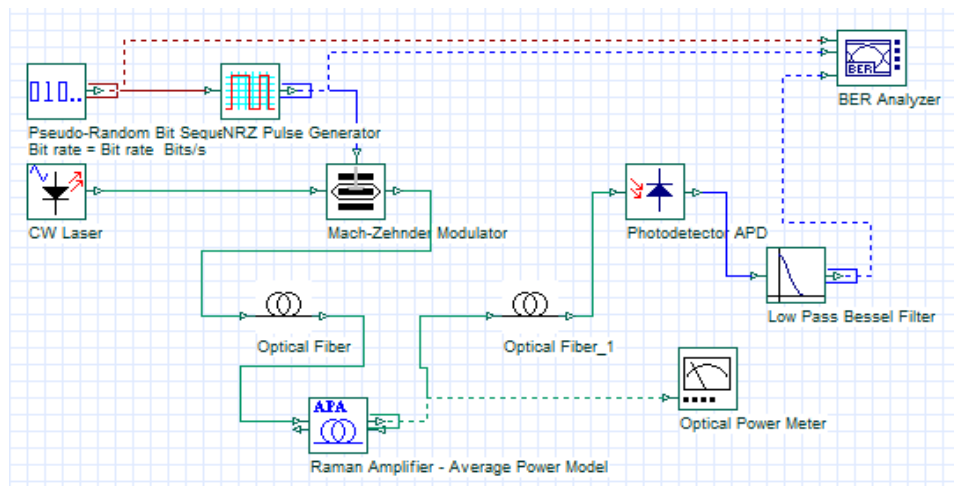

Figure F.7. Optical Fiber System Scheme with FRA InlineAmplifier

\section{RESULT AND DISCUSSION}

The result of SOA is depicted in Figure F.8. The BER may be analyzed using stochastic computer simulations. If a simple transmission channel model and data source mode is assumed, the BER may also be calculated analytically. In absence of available device for BER analysis, OTDR has been used for checking the signal loss in fiber optics and the OptiSystem for analyzing the BER. The circuit diagram of Figure F.6 has been used to do this analysis. BER is found in SOA where $1350 \mathrm{~nm}$ wavelength with $1 \mathrm{~mW}$ input power is better than the others since it has higher energy rather than $1470 \mathrm{~nm}$ and $1560 \mathrm{~nm}$, so that BER oscillation depends on energy. However, after about $150 \mathrm{~km}, \mathrm{BER}$ value increases. It is not surprising when the distance is long then the error will come, this is due to attenuation of geometry length where it can operate either low or high energy sources corresponding to wavelength source. Even at $120 \mathrm{~km}$, the highest BER is achieved for $1350 \mathrm{~nm}$ (highest energy). This is the weaknesses of SOA characteristics.

Although these data are unknown source of factors but improving BER can be detected by choosing strong signal strength, a slow and robust modulation scheme or line coding scheme, or by applying channel coding schemes such as redundant forward error correction codes.

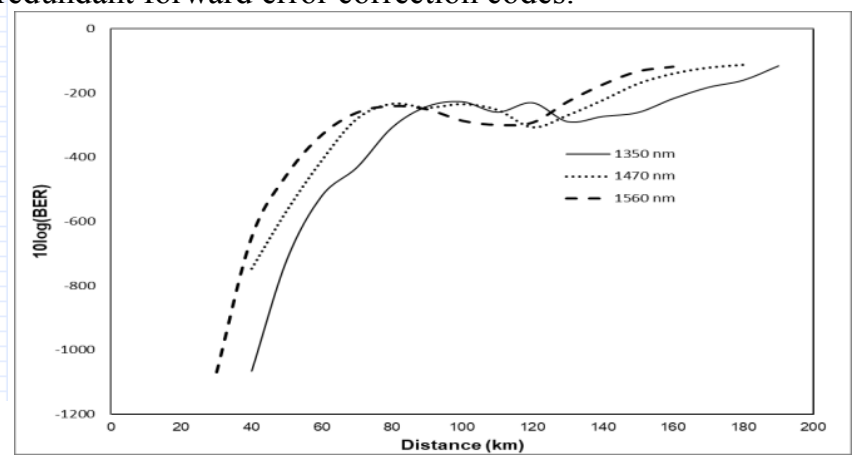

Figure F.8. BER for SOA 
As well as BER of SOA, Q-factor of SOA goes down as a distance is increased. But, Q-factor for $1350 \mathrm{~nm}$ (highest energy) even is less decay rather than the others. Although the decay trends are stable beginning from $80 \mathrm{~km}$ similar to a constant Q-factor. At $140 \mathrm{~km}$ the energy source is not good enough to maintain the oscillation source hence the decay goes down near linear including $1350 \mathrm{~nm}$ and keep maintaining to reduce it slowly.

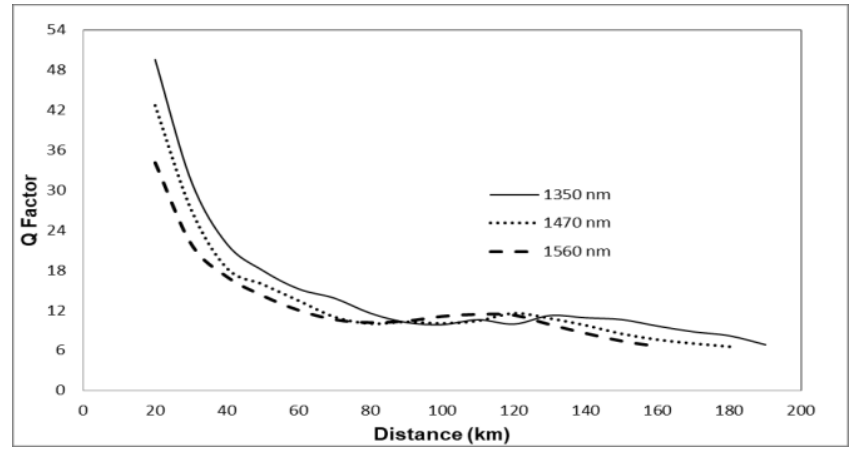

Figure F.9. Q-factor of SOA

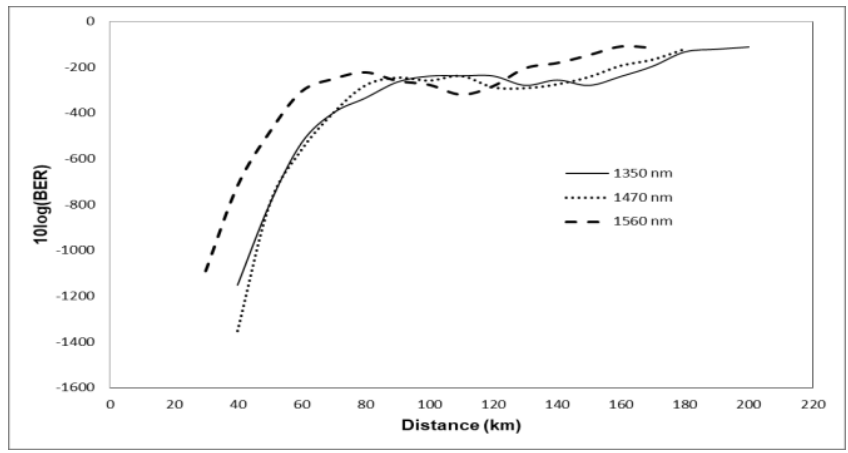

Figure F.10. BER for FRA

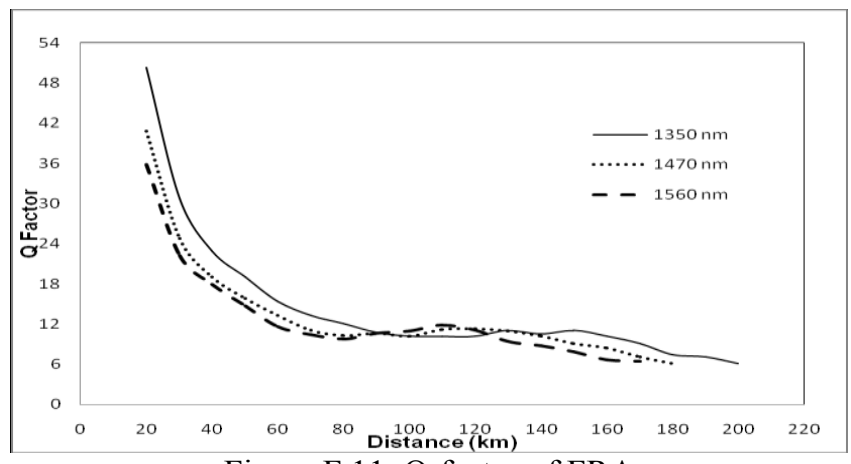

Figure F.11. Q-factor of FRA

Unlike SOA, FRA has good performance for both BER and Q-factor. The result of FRA for BER and Q-factor is depicted in Figure F.10 and F.11. BER is less fluctuated and more stable for higher energy of $\mathrm{E}=\mathrm{hf}$, where $\mathrm{f}$ is frequency source, $\mathrm{h}$ is Planck constant and $\mathrm{E}$ is energy. From 60 until $140 \mathrm{~km}, \mathrm{BER}$ is nearly constant for various wavelength source, hence this BER is better than SOA. Q-factor is faster for a stable condition at higher energy at $80 \mathrm{~km}$ and continues after $160 \mathrm{~km}$. This performance shows that at wavelength of $1350 \mathrm{~nm}$, the dispersion is more than wavelength of $1560 \mathrm{~nm}$ but $\mathrm{Q}$ factor osicillation is low.

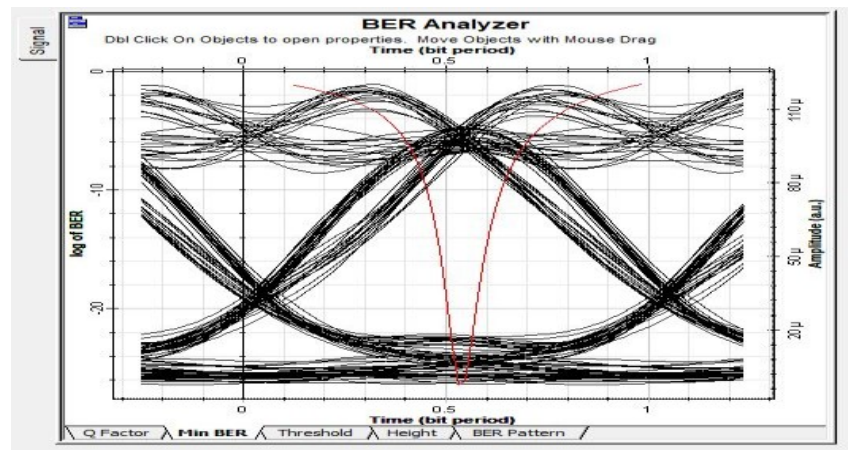

(a)

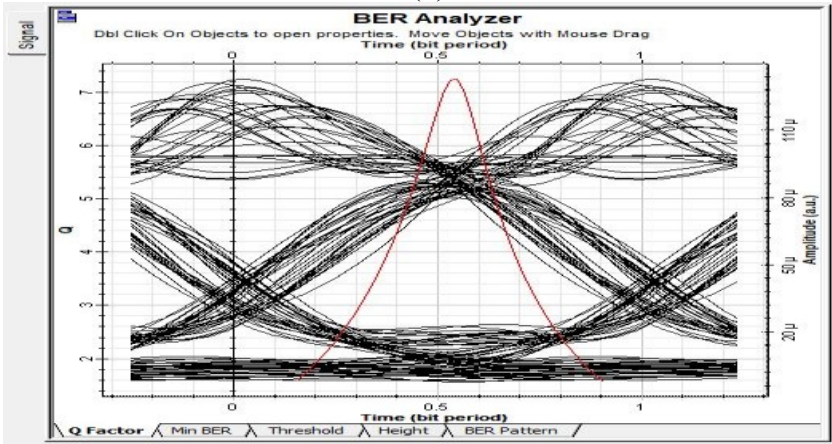

(b)

Figure F.12. (a). $1350 \mathrm{~nm}, 85 \mathrm{~km}$, (b).1560nm, $85 \mathrm{~km}$

Figure F.12. is an eye patern diagram with no amplifier. It is generally seen that the amplitude and bit error rate differ greatly at the lower distances at (a) than (b). The weakness of the unfocused amplitude due to the power and geometry of the far wave from the wave source. The red line facing at below is BER and the red line facing a top is Q-factor.

In Figure F.13, both wavelengths at $160 \mathrm{~km}$ distance have a low Q-factor especially at low wave energy. SOA function is very effective at a distance less than $100 \mathrm{~km}$, but at the peak of $160 \mathrm{~km}$, the wave amplitude is not focused anymore. Amplitude is affected by distance even if SOA is used.

Figure F.14 is somewhat different than SOA. FRA has a more effective BER and Q-factor. At a distance of less than $90 \mathrm{~km}$ the very sharp eye pattern at $1350 \mathrm{~nm}$ wavelength is almost equal to the $1560 \mathrm{~nm}$ wavelength. The value of FRA at a distance of $170 \mathrm{~km}$ is still more effective than on SOA values both at $1350 \mathrm{~nm}$ and at $1560 \mathrm{~nm}$. However at $1560 \mathrm{~nm}, \mathrm{BER}$ is more sharp as Q-factor aa well. BER and Q-factor at 1560 is higher so that the amplifier function is weak especially at great distances. 


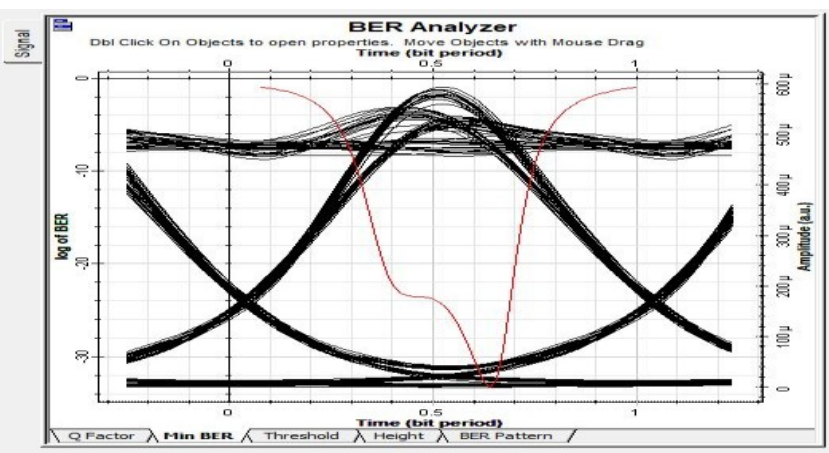

(a)

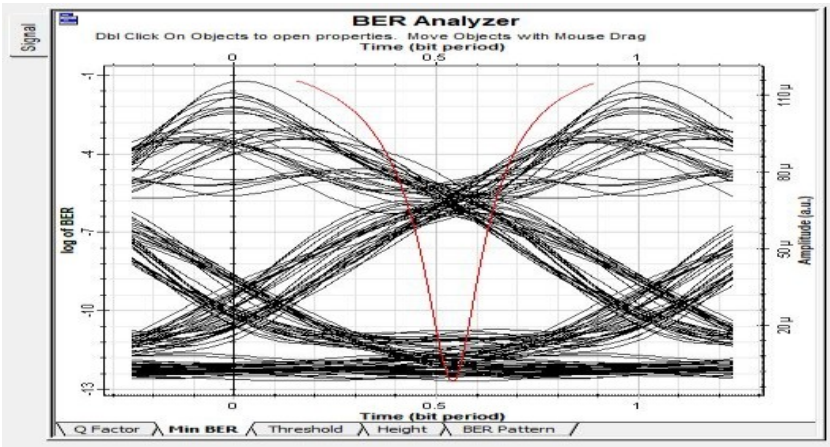

(b)

Figure F.13. (a). SOA (BER and Q-factor) $1350 \mathrm{~nm}, 80 \mathrm{~km}$,

(b). SOA (BER and Q-factor) $1560 \mathrm{~nm}, 160 \mathrm{~km}$

\section{CONCLUSION}

Although the optical amplifier can maintain the signal along the trajectory of waveguide, several amplifiers still have problems. Both SOA and FRA have advantages and disadvantages. Using the simulation application, both amplifiers are succesfully designed and compared by inline amplifiers. The results described that the transmission distance of the FRA is much farther than the SOA shown by BER and Q-factor. However, this FRA system has higher power consumption when compared to the SOA system.

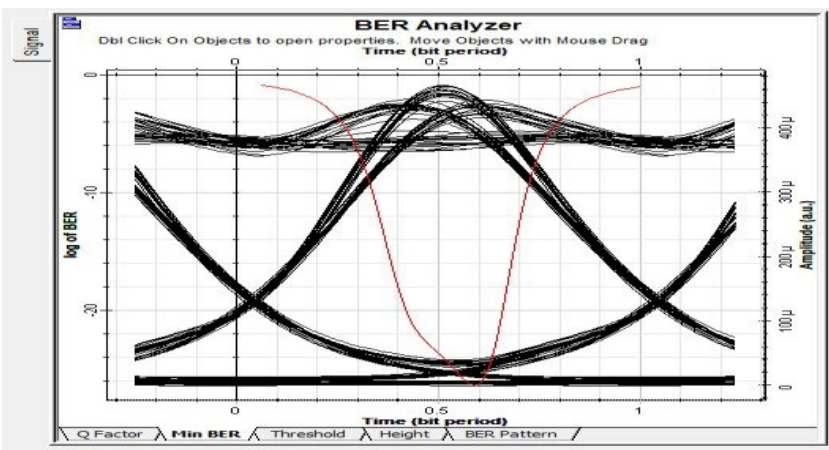

(a)

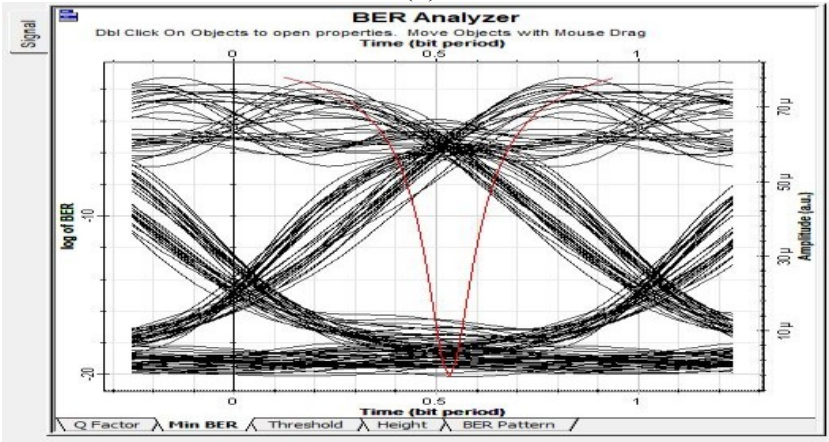

(b)

Figure F.14. (a) Raman (BER, Q-factor) 1350nm, 90km, (b). Raman (BER and Q-factor) $1350 \mathrm{~nm}, 170 \mathrm{~km}$

\section{REFERENCES}

[1] Karimah Z N, Hambali A, Suwandi. Analisis Perbandingan Kinerja Mach-Zehnder berdasarkan Ragam Format Modulasi pada Jaringan FTTH. Jurnal ELKOMIKA. 2017; 5(1): 73-92

[2] Leuthold J, Bonk R, Vallaitis T, Marculescu A, Freude W, Meuer C, Bimberg D, Brenor R, Lelarge F, Duan G H. Linier and Nonlinier Semiconductor Optical Amplifiers. Conference on Optical Fiber Communication (OFC/NFOEC), collocated National Fiber Optic Engineers Conference. San Diego. 2010

[3] Sun H, Wang Q, Dong H, Dutta N K. XOR Performance of a Quantum Dot Semiconductor Optical Amplifier based Mach-Zehnder Interferometer. Optics Express. 2005; 13(6): 1892-1899

[4] Hamie A, Sharaiha A, Guegan M, Pucel B. All-Optical Logic NOR Gate Using Two-Cascaded Semiconductor Optical Amplifiers. IEEE Photonics Technology Letters. 2002; 14(10): 1439-1441

[5] Kanellos G T, Petrantonakis D, Tsiokos D, Bakopoulos P, Zakynthinos P, Pleros N, Apostolopoulos D, Maxwell G, Poustie A, Avramopoulos H. All-Optical 3R Burst-Mode Reception at $40 \mathrm{~Gb} / \mathrm{s}$ Using Four Integrated MZI Switches. Journal of Lightwave Technology. 2007; 25(1): $184-192$

[6] Dong J, Zhang X, Fu S, Xu J, Shum P, Huang D. Ultrafast All-Optical Signal Processing Based on Single Semiconductor Optical Amplifier. IEEE Journal of Selected Topics in Quantum Electronics. 2008; 14(3): 770-778

[7] Bromage J. Raman Amplification for Fiber Communications Systems. IEEE Journal of Lightwave Technology. 2004; 22(1): 79-93. 